\title{
Is Autoverification of Reports the Need of the Hour in Clinical Chemistry Laboratory?
}

\author{
${ }^{1}$ Swati Rajput, ${ }^{2}$ Shilpa Jain
}

\begin{abstract}
Introduction: Clinical laboratories need to respond to the challenges of reducing manpower and decreasing the turnaround time by simplifying the process of report release. This can be achieved by building an autoverification (AV) system in the clinical chemistry laboratory.
\end{abstract}

\section{Aims and objectives:}

- To establish the criteria for AV.

- To know the proportion of tests or the sample load which is autoverifiable.

- To compare advantages and disadvantages of AV.

Materials and methods: A descriptive observational study was conducted on test results in clinical chemistry laboratory of Sir Sayajirao General Hospital and Medical College, Vadodara, India, from April 15 to July 15, 2016. At the end of the day, all reports were analyzed and the ones autoverifiable along with their percentage were calculated.

Results and observations: In our laboratory, percentage of autoverifiable reports was found to be between 71 and $89 \%$ for various parameters.

Conclusion: Although AV is a complex task, the outcome is absolutely worth the effort. Autoverification, if properly integrated into laboratory information system (LIS), is very fast as compared with the manual verification done by skilled laboratory medical biochemists. Implementation of AV not only decreases the turnaround time but also leads to more consistent verification of test results as all results go through the same process before being released.

Keywords: Autoverification, Laboratory information system, Middleware design, Quality control.

How to cite this article: Rajput S, Jain S. Is Autoverification of Reports the Need of the Hour in Clinical Chemistry Laboratory? Indian J Med Biochem 2018;22(1):56-60.

Source of support: Nil

Conflict of interest: None

\section{INTRODUCTION}

Autoverification is the process by which patients' test results generated from interfaced instruments are sent

\footnotetext{
${ }^{1}$ Student, ${ }^{2}$ Professor and Head

1,2Department of Biochemistry, Sir Sayajirao General Hospital and Medical College, Vadodara, Gujarat, India

Corresponding Author: Swati Rajput, Student, Department of Biochemistry, Sir Sayajirao General Hospital and Medical College, Vadodara, Gujarat, India, Phone: +917096938527 e-mail: career655@gmail.com
}

to LIS or middleware, where they are compared against the laboratory-defined acceptance parameters. ${ }^{1}$ Middleware is the information software installed between the LIS and the instrument as shown in Figure 1. It delivers information, such as the test orders from the LIS to the instrument and the test results of the instrument back to the LIS. ${ }^{2}$ Since the workload in clinical chemistry laboratory is very high, especially in a tertiary care center, it is a mammoth task to go through each examination and its reporting. In this scenario, $\mathrm{AV}$ can greatly reduce the time and effort of laboratory staff and final verification signatories, so that they can concentrate on other important aspects of laboratory like quality monitoring. Every examination in a patient sample performed by medical laboratory is to be followed by postexamination verification procedure. Even the most primitive biochemistry laboratory performs rudimentary verification of laboratory examination results just before printing the report or delivering it to the patient. ${ }^{1,2}$

Use of computer for postexamination verification called AV is an answer to the problem of verification of large number of test results produced by modern-day busy clinical chemistry laboratories. When the sample load is high, competent medical biochemists who are constantly verifying the reports are also prone to human error. To meet all these demands and to decrease the human error, a concept of AV is put forward.

There are several software tools available for AV which can be used in a clinical chemistry laboratory. SMART TESTS is an AV design/software technique that can be used to build an AV system. ${ }^{3}$ SMART TESTS is an acronym that stands for Scope, Master algorithm template, Analyzer-specific algorithm template, Reagentspecific algorithm, Tool selection and implementation, Translation of algorithms into rules, Exceptions review process, Substantiation (validation), Train, and Scale. ${ }^{3}$ Other software tools that can be used are Linux operating system, html programming, Apache server, and PHP (Hypertext preprocessor). ${ }^{4}$ Minimum requirements for the software tools to build AV algorithm include the following ${ }^{3}$ :

- Ability to use multiple data elements in an unrestricted fashion.

- Application of the algorithm in real time. 


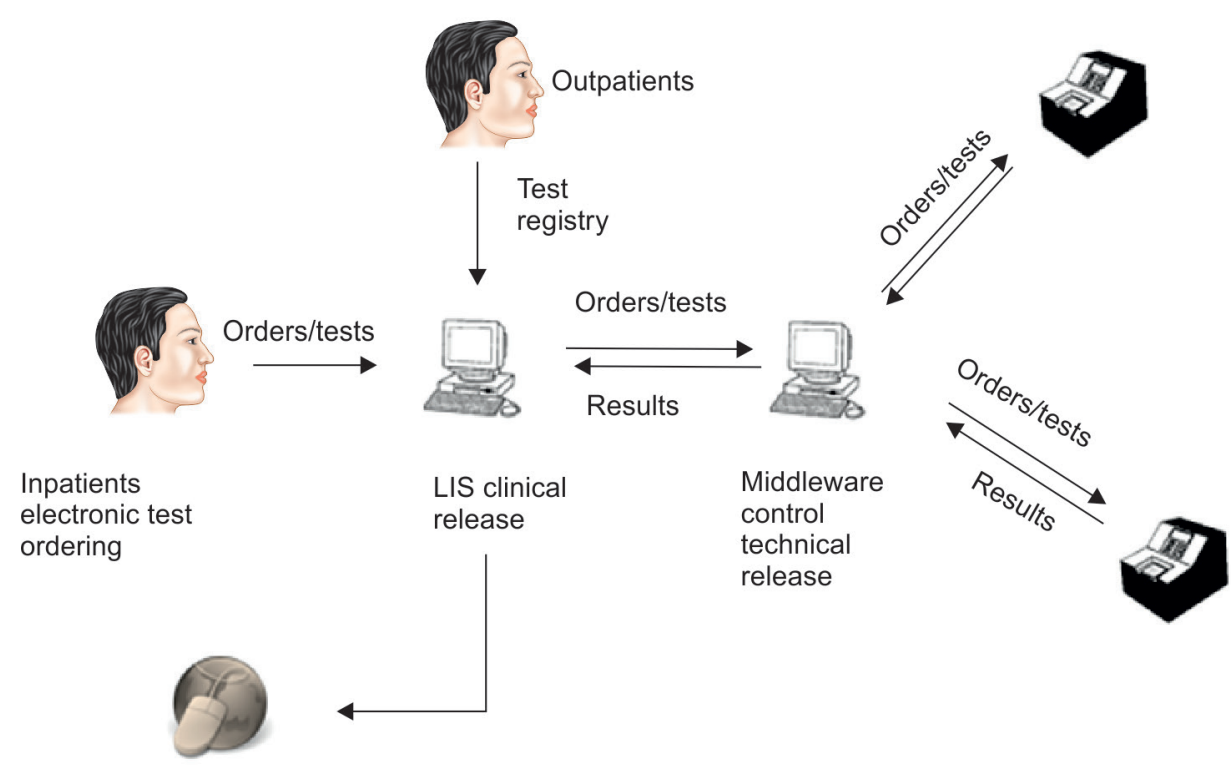

Fig. 1: The middleware and $\mathrm{LIS}^{2}$

\begin{tabular}{|c|c|c|c|c|c|c|c|}
\hline \multicolumn{4}{|c|}{ Pre examination } & \multicolumn{2}{|c|}{ Examination } & \multicolumn{2}{|c|}{ Post examination } \\
\hline 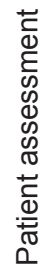 & 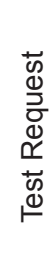 & 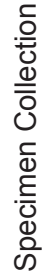 & 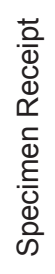 & 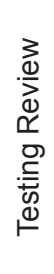 & 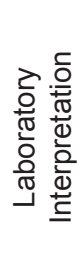 & 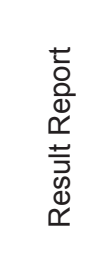 & 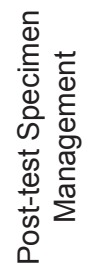 \\
\hline
\end{tabular}

Fig. 2: The path of workflow ${ }^{6}$

- Ability of the laboratory to define and implement changes to algorithm quickly and easily.

- Retrieval of selected information from multiple data sources (e.g., pharmacy, instrument results, other laboratory data).

- Flexible user interface that provides laboratorydefined information on the $\mathrm{AV}$ process in real time.

Clinical and Laboratory Standards Institute (CLSI), Wayne, United States, in 2006 had released the CLSI document AUTO10-A guidelines for AV for clinical laboratory test results. This document allows each laboratory to easily design, implement, validate, and customize rules for $\mathrm{AV}$ based on the needs of its patient population. ${ }^{5}$ Through utilization of this approach, the users will not only be able to ensure compliance with regulatory agencies (where acceptable by law), but also effectively develop and establish measures to ensure that all aspects related to quality are maintained. The CLSI document GP26-Application of a Quality Management System Model for Laboratory Services defines a clinical laboratory path of work flow which is necessary to deliver the results. ${ }^{6}$ This consists of three sequential processes: Preexamination, examination and postexamination. All clinical laboratories must follow these processes to deliver quality laboratory services. Figure 2 shows the path of workflow. ${ }^{6}$ There are certain rules or preconditions for AV like quality control, delta check, critical value, and consistency check. The quality control for the parameters that need to be assessed should be within the laboratory's acceptable limit. Any instrumental error, if present, should be eliminated. Delta checks are investigated internally to rule out mislabeling, topographical error or any possible analytical error. Critical values, if present, will hinder the process of AV and the report must be manually verified. ${ }^{7}$

Basic schematic representation of AV process can be seen in Figure 3. The figure shows that preanalytical, analytical, and postanalytical data go to AV engine, where they pass through the rules one by one. If all rules are successfully met, then autoverified results are directly sent to LIS. ${ }^{4}$

\section{MATERIALS AND METHODS}

A descriptive observational study was carried out on results of samples analyzed in clinical chemistry laboratory of Sir Sayajirao General Hospital and Medical 
Preanalytical information

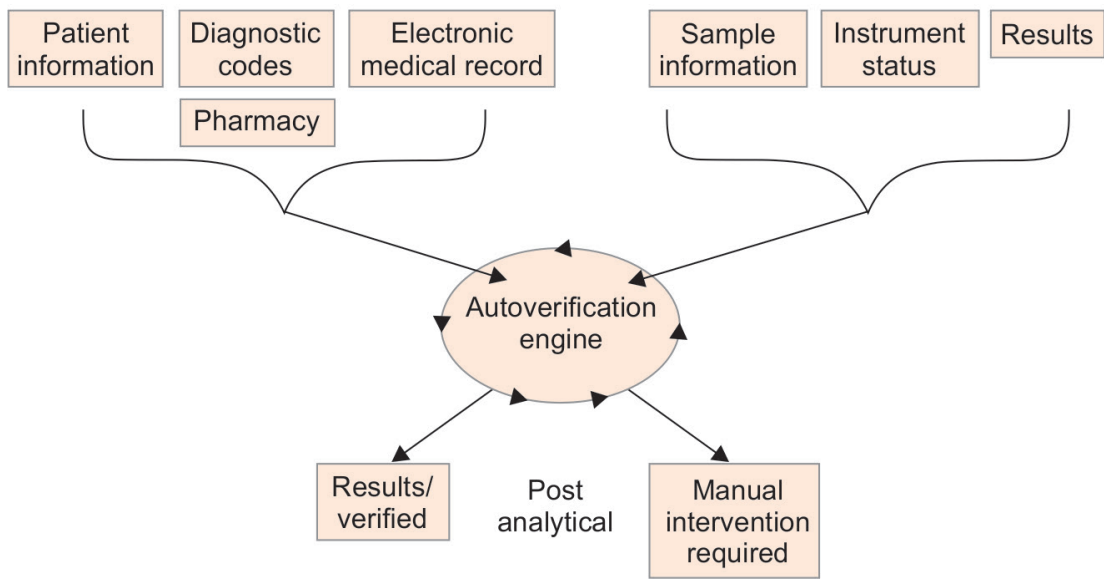

Fig. 3: Schematic representation of $A V^{4}$

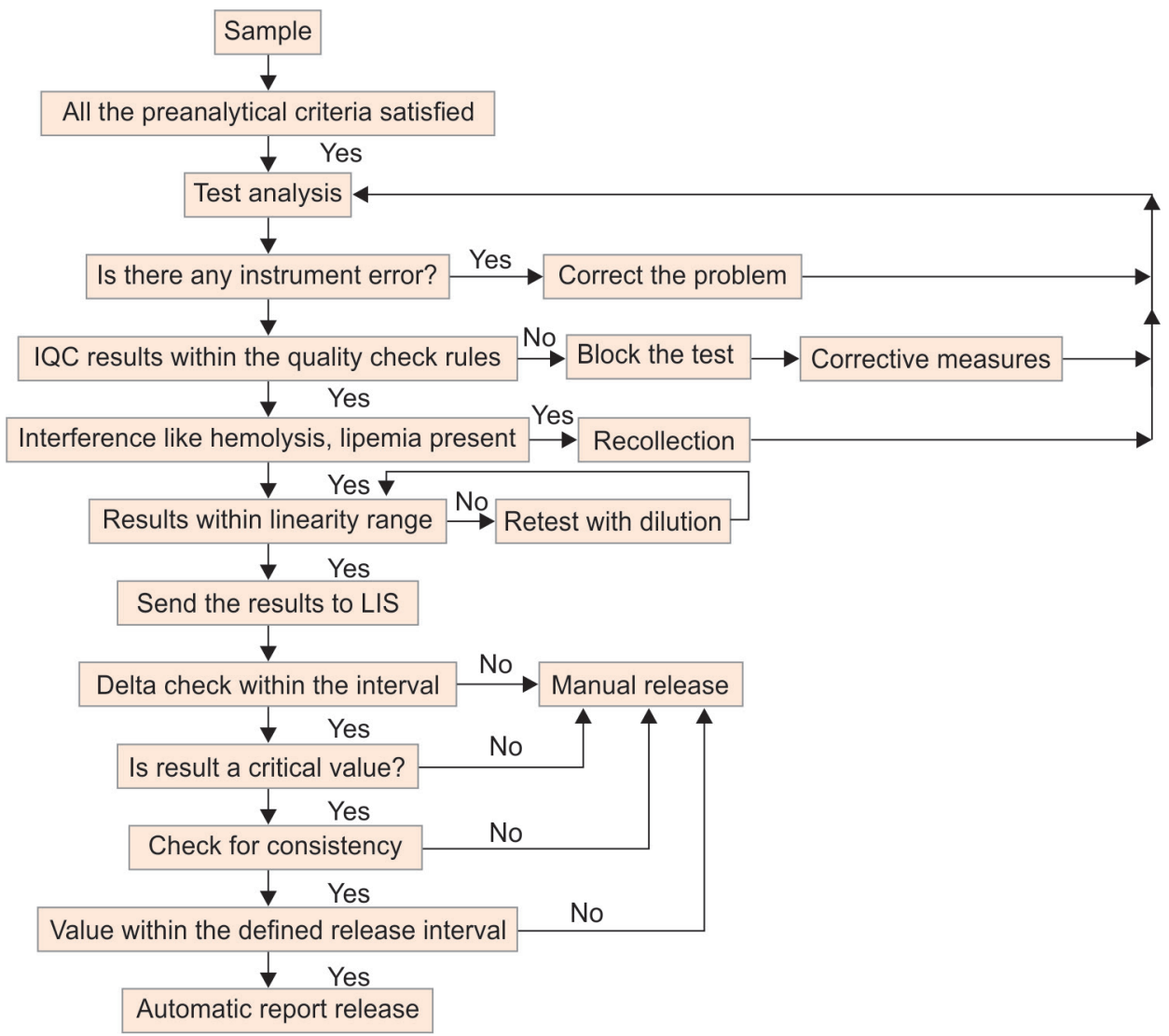

Fig. 4: A proposed model of algorithm for AV

College, Vadodara, from April 15 to July 15, 2016. Based on the rules for $\mathrm{AV}$ which include delta check, critical value, consistency check, and linearity, we designed an algorithm as shown in Figure 4. At the end of day, all results for the six routine parameters, glucose, total bilirubin, creatinine, urea, total protein, and albumin were checked manually as per the algorithm and the ones fulfilling the criteria of AV were noted as autoverifiable and the rest were labeled as "manual verification requiring reports."

To know the number of man-hours spent on verification of reports, we noted down the number of final and interim signatories in the department and the number of tests performed. Daily duty schedule was noted and individual faculty's time spent on the verification was also noted from individual faculty's $\log$ in and log out time.

\section{Statistical Analysis}

After the collection of the data for 3 months, the results were entered in the Microsoft Excel software and the percentage of the reports autoverifiable and man-hours spent on AV were calculated. 
Is Autoverification of Reports the Need of the Hour in Clinical Chemistry Laboratory?

\section{RESULTS AND OBSERVATIONS}

In this study period, from April 15 to July 15, 2016, total number of samples received in our clinical chemistry laboratory was 37,458 and total number of tests done in these 3 months was 107,789. In our laboratory, all test results are verified manually before the report is released. Table 1 shows our observations, out of the total tests done during this period, the six common parameters, i.e., glucose, urea, total bilirubin, creatinine, total protein, and albumin constituted 74,212 tests. Out of these 74,212 tests, the number of tests done for glucose was 13,898 , for urea, it was 19,865 , for total bilirubin, it was 18,545 , for creatinine, it was 12,966 , for total protein, it was 4,449 , and for albumin, it was 4,489.

Out of total tests done for each of the six parameters, the number of reports autoverifiable for glucose was $12,450 / 13,898$, for urea, it was $17,240 / 19,865$, for total bilirubin, it was $14,234 / 18,545$, for creatinine, it was $10,865 / 12,966$, for total protein, it was $3195 / 4449$, and for albumin, it was 3215/4489.

The proportion of autoverifiable reports for each of these parameters, i.e., glucose, urea, total bilirubin, creatinine, total protein, and albumin was $89.5,86.7,76.7,83.8,71.8$ and $71.6 \%$ respectively, as shown in Table 1 and Graph 1 .

Table 1: The percentage of reports which are autoverifiable and those requiring manual verification

\begin{tabular}{lllll}
\hline & $\begin{array}{l}\text { Number } \\
\text { Number }\end{array}$ & $\begin{array}{l}\text { Nof of } \\
\text { of tests } \\
\text { outoverifiable }\end{array}$ & $\begin{array}{l}\text { requiring } \\
\text { manual }\end{array}$ \\
parameter & done & autoverifiable reports & verification \\
\hline Glucose & 13,898 & 12,450 & 89.5 & 10.5 \\
Urea & 19,865 & 17,240 & 86.7 & 13.3 \\
Total bilirubin & 18,545 & 14,234 & 76.7 & 23.3 \\
Creatinine & 12,966 & 10,865 & 83.8 & 16.2 \\
Total protein & 4,449 & 3,195 & 71.8 & 28.2 \\
Albumin & 4,489 & 3,215 & 71.6 & 28.4 \\
\hline Total & 74,212 & 61,199 & 82.5 & 17.5 \\
\hline
\end{tabular}

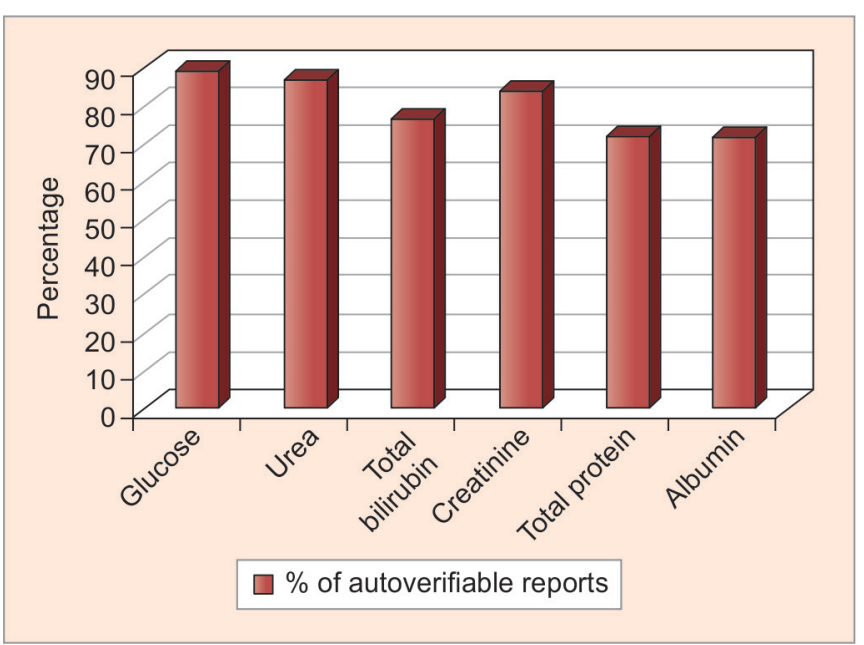

Graph 1: The percentage of autoverifiable reports
Table 2: The man-hours spent on AV per day

\begin{tabular}{llll}
\hline $\begin{array}{l}\text { Total number } \\
\text { of final }\end{array}$ & $\begin{array}{l}\text { Total number } \\
\text { of interim } \\
\text { signatories }\end{array}$ & $\begin{array}{l}\text { Average time } \\
\text { signatories }\end{array}$ & $\begin{array}{l}\text { Man hours spent } \\
\text { on verification } \\
\text { sper day }\end{array}$ \\
\hline 9 & 4 & 4.3 hours & 34.4 hours \\
\hline
\end{tabular}

In our laboratory, there are nine final signatories and four interim signatories for report verification. Total numbers of samples per day are 500 to 600 and total numbers of tests performed each day are around 2000. Table 2 shows the average time spent and the man-hours spent on manual verification per day.

\section{DISCUSSION}

In this study, we found that 70 to $90 \%$ of the reports are autoverifiable; maximum percentage of autoverifiable reports was seen in the case of glucose followed by urea and creatinine. Total protein and albumin showed nearly the same percentage of autoverifiable reports. According to a study published by Torke et $\mathrm{al}_{,}^{8}$ as part of an ongoing modular laboratory automation plan at John H. Stroger, Jr. Hospital of Cook County (formerly Cook County Hospital), Chicago, the rate of AV was $73 \%$. Our results are similar to this study. Shih et al ${ }^{7}$ reported the $\mathrm{AV}$ rate to be as high as $95 \%$ for all the test results, which is higher than the findings of this study. This difference could be because in their laboratory, acceptable range for the delta check and limit check for each test item might be different from ours.

In this study, we found that for verification of 1500 to 2000 tests per day, 34.4 man-hours are being spent, which can be reduced to approximately 10 man-hours by AV. This time that is saved can be spent for academic activities, quality improvement in the laboratory, research work, and introduction of new tests.

The role of AV is that it not only detects errors in the test results, but also leads to improvement in patients' clinical care. For example, results in the delta check falling outside the acceptable range could imply a significant change in the patients' condition. In such cases, the laboratory would alert the physician so that the patient can receive appropriate timely treatment. ${ }^{7,9}$

There are several advantages of $\mathrm{AV}$, such as improved consistency of reporting, appreciable reduction of errors, reduction in the amount of labor required for the verification of reports, decreased turnaround time, more organized work flow, and increased staff morale with less fatigue and stress. Despite the advantages of AV, there are potential negatives that can arise. The installation and validation of $\mathrm{AV}$ are time-consuming, costly, and require trained personnel. The other most common problem is the interruption of network, LIS, middleware, and the interfaces between these systems. The other 
risk with $\mathrm{AV}$ and indeed with increased automation in general is the reduction of staff (both in number of staff and the risk of level of training and experience) to such a degree that the staff cannot handle downtimes or other challenges without severe compromise of turnaround time. ${ }^{10}$ Quality control system is usually not connected with AV system of the middleware; hence, the results of QC need to be monitored manually. For this reason, the QC system should be integrated with the AV system, so that when $\mathrm{QC}$ failure occurs, the $\mathrm{AV}$ of the given test item automatically stops. 7,11

There are certain limitations to our study. The AV is not yet installed in our laboratory due to limited technical resources to dedicate to the project, lack of technical experience, and absence of established AV implementation process. This pilot study was done to theoretically anticipate how AV system, if installed, could benefit our laboratory.

\section{CONCLUSION}

Although installation of AV is a complex and timeconsuming project, the payoff is absolutely worth the effort. The task of verification is completed by computer at a much faster rate with minimal need for human intervention. The AV is also very useful because day by day, clinical laboratories are facing greater challenges in terms of maintaining higher accuracy and precision and at the same time reducing the turnaround time. It is not only a need of present working scenario but also a boon for all clinical laboratories.

Autoverification, if properly validated for each and every possible outcome, is fault-proof as compared with manual verification, which may lead to considerable omission of results. To be successful, it should be implemented in stages after the laboratory is comfortable with the instrument systems. Finally, the best path to successful implementation is to start simply with few tests and then build the rules in stages and always one should be sure of the quality management system before attempting to set up AV.

\section{REFERENCES}

1. Bowen, R. Autoverification in clinical chemistry: from theory to practice. Palo Alto (CA): Stanford University; 2011. pp. 1-12.

2. Feitosa MS, Bucker DH, Santos SM, Vasconcellos LS. Implementation of criteria for automatic release of clinical chemistry test results in a laboratory at an academic public hospital. J Bras Patol Med Lab 2016 May-Jun;52(3):2-8.

3. Marquardt B. A step by step process to $95 \%$ autoverification. Cap Today 2015 Dec;1:1-6.

4. Nikunj, PR. Development and implementation of autoverification algorithm for observed values of examinations in clinical chemistry. MD [Dissertation]. Surat: Veer Narmad South Gujarat University; 2013.

5. CLSI. Autoverification of clinical laboratory test results; approved guidelines. CLSI document AUTO10-A. Wayne (PA): Clinical and Laboratory Standards Institute; 2006.

6. CLSI/NCCLS. Application of a quality management system model for laboratory services; approved guideline. 3rd ed. NCCLS document GP26-A3. Wayne (PA): NCCLS; 2004.

7. Shih MC, Change HM, Tien N, Hsiao CT, Peng CT. Building and validating an autoverification system in the clinical chemistry laboratory. Lab Med 2011 Nov;42(11):668-673.

8. Torke N, Boral L, Nguyen T, Perri A, Chakrin A. Process improvement and operational efficiency through test results autoverification. Clin Chem 2005 Dec;51(12):2406-2408.

9. Person, NB. Introduction to autoverification. Global Product Education. 2011. pp. 1-32.

10. KrasowskiMD,Davis SR, DreesD, Morris C,Kulhavy J,CroneC, Bebber T, Clark I, Nelson DL, Teul S, et al. Autoverification in a core clinical chemistry laboratory. J Pathol Inform 2014 Mar;5(1):13.

11. Duca DJ. Autoverification in a laboratory information system. Lab Med 2002 Jan;33(1):21-25. 